\title{
Gender-Related Difference in D-Dimer Level Predicts In-Hospital Heart Failure after Primary PCI for ST-Segment Elevation Myocardial Infarction
}

\author{
Li Li, ${ }^{1}$ Wei Wang, ${ }^{1}$ Tai Li, $^{2}$ Ying Sun, ${ }^{1}$ Yanjun Gao, ${ }^{3}$ Lin Wang, ${ }^{4}$ and Heng-Chen Yao $\mathbb{D}^{1}$ \\ ${ }^{1}$ Department of Cardiology, Liaocheng People's Hospital, Liaocheng 252000, China \\ ${ }^{2}$ Department of Nursing, Liaocheng Vocational \& Technical College, Liaocheng 252000, China \\ ${ }^{3}$ Department of Clinical Laboratory, Liaocheng People's Hospital, Liaocheng 252000, China \\ ${ }^{4}$ Cardiologic Color Doppler Room, Liaocheng People's Hospital, Liaocheng 252000, China
}

Correspondence should be addressed to Heng-Chen Yao; yaohc66@hotmail.com

Received 29 April 2021; Accepted 23 July 2021; Published 11 August 2021

Academic Editor: Paulina Dumnicka

Copyright (c) $2021 \mathrm{Li} \mathrm{Li}$ et al. This is an open access article distributed under the Creative Commons Attribution License, which permits unrestricted use, distribution, and reproduction in any medium, provided the original work is properly cited.

\begin{abstract}
Aims. The prognostic value of plasma D-dimer in patients with coronary artery disease (CAD) remains controversial. The study is aimed at investigating the relationship between plasma D-dimer levels and in-hospital heart failure (HF) in ST-segment elevation myocardial infarction (STEMI) patients who underwent primary percutaneous coronary intervention (pPCI). Methods. STEMI patients who underwent pPCI were enrolled in this study. Venous blood samples were collected from patients on admission before pPCI procedure. The study endpoint was the occurrence of in-hospital HF. The participants were divided into two groups according to plasma D-dimer levels and further compared baseline $\mathrm{D}$-dimer levels between male and female. Logistic regression and receiver operating characteristic (ROC) curves were performed to evaluate the relationship of D-dimer and inhospital HF. Results. A total of 778 patients were recruited in the study, of which 539 (69.3\%) patients had normal D-dimer levels $(\leq 0.5 \mathrm{mg} / \mathrm{L})$ while $239(30.7 \%)$ had increased D-dimer levels $(>0.5 \mathrm{mg} / \mathrm{L})$. The female patients have higher D-dimer levels and higher incident rate of in-hospital HF than that in male patients $(p<0.001)$. The multivariate logistic regression model revealed that D-dimer was an independent predictor for in-hospital HF in overall population (adjusted odds ratio [OR]: 1.197, 95\% CI: 1.003-1.429, and $p=0.046$ ) and female patients (adjusted OR: 1.429, 95\% CI: 1.083-1.885, and $p=0.012$ ). Conclusion. Increased plasma D-dimer levels were an independent risk factor for incidence of in-hospital HF in STEMI patients who underwent pPCI, especially in female patients, which provides guidance for clinicians in identifying patients at high risk of developing HF and lowering their risk.
\end{abstract}

\section{Introduction}

D-dimer is a biomarker of thrombosis and hypercoagulability; it can be measured in plasma or whole blood $[1,2]$. Elevated plasma levels of $\mathrm{D}$-dimer have been proven to be associated with subsequent thrombotic events and are extensively used to diagnose venous thromboembolism (VTE) [35]. Previous studies showed that D-dimer levels could predict adverse outcomes, including fatal events in patients with coronary heart disease (CHD), in terms of both short-term outcomes and long-term follow-up [5-10].
Heart failure (HF) following myocardial infarction (MI) is common and may develop during hospitalization; it may even be present on admission [11]. Furthermore, HF is the strongest predictor of death and has a great implication for treating patients with acute MI, especially women [12]. Previous studies demonstrated that D-dimer levels were more predictive for cardiac events in patients with acute MI; however, HF was not included $[8,13,14]$. To date, the role of D-dimer level in predicting in-hospital HF in patients with STsegment elevation MI (STEMI) is not clear, particularly after primary percutaneous coronary intervention (pPCI). 


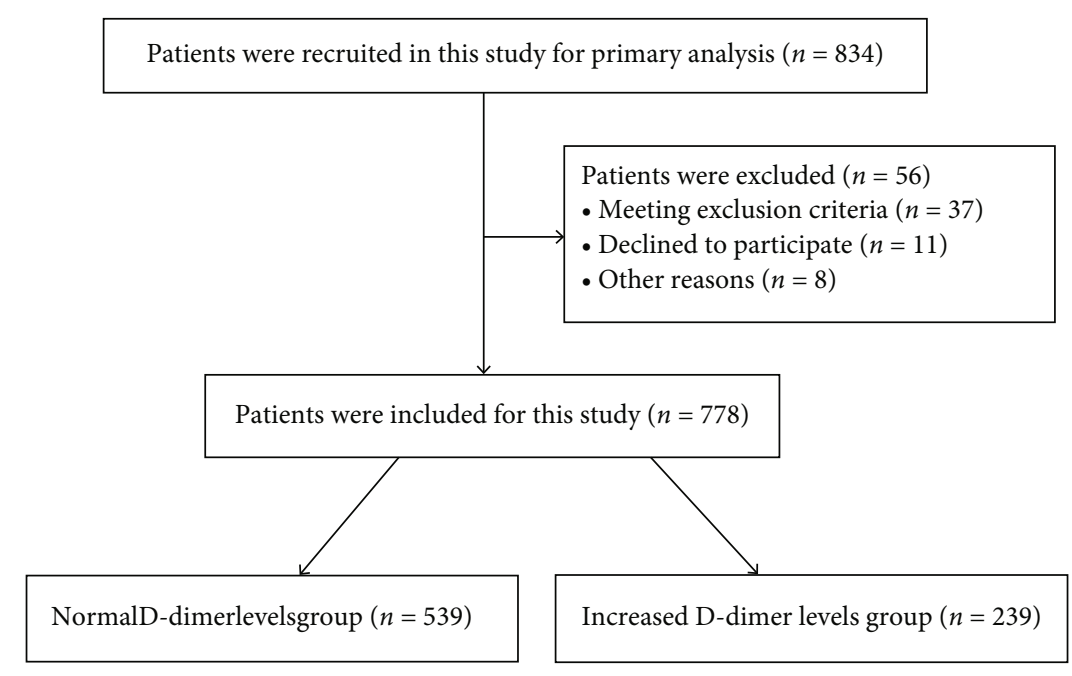

FIGURE 1: The flowchart of studied patients.

TABLE 1: Basic characteristics of overall population by D-dimer level.

\begin{tabular}{|c|c|c|c|}
\hline Variable & $\begin{array}{c}\mathrm{D}-\operatorname{dimer} \leq 0.5 \\
\mathrm{mg} / \mathrm{L} \\
n=539\end{array}$ & $\begin{array}{c}\mathrm{D}-\text { dimer }>0.5 \\
\mathrm{mg} / \mathrm{L} \\
n=239\end{array}$ & $p$ value \\
\hline Age (years) & $61(17)$ & $65(16)$ & $<0.001$ \\
\hline Smoking, $n(\%)$ & $302(56.0)$ & $114(47.7)$ & 0.032 \\
\hline $\begin{array}{l}\text { Hypertension, } n \\
\text { (\%) }\end{array}$ & $290(53.8)$ & $128(53.5)$ & 0.949 \\
\hline $\mathrm{DM}, n(\%)$ & $141(26.2)$ & $63(26.4)$ & 0.953 \\
\hline Heart rate (bpm) & $76(20)$ & $78(20)$ & 0.233 \\
\hline $\begin{array}{l}\text { Hemoglobin } \\
(\mathrm{g} / \mathrm{dL})\end{array}$ & $144(20)$ & $139(24)$ & $<0.001$ \\
\hline $\begin{array}{l}\text { WBC count } \\
(\times 109 / \mathrm{L})\end{array}$ & $9.67(3.83)$ & $9.97(4.30)$ & 0.281 \\
\hline PLT $(\times 109 / \mathrm{L})$ & $231(74)$ & $221(85)$ & 0.064 \\
\hline $\mathrm{Cr}(\mu \mathrm{mol} / \mathrm{L})$ & $62(21)$ & $64(19)$ & 0.002 \\
\hline $\mathrm{TC}(\mathrm{mmol} / \mathrm{L})$ & $4.78(1.32)$ & $4.64 \pm 0.99$ & 0.033 \\
\hline TG $(\mathrm{mmol} / \mathrm{L})$ & $1.52(1.26)$ & $1.35(1.00)$ & 0.006 \\
\hline $\mathrm{EF}(\%)$ & $50(9)$ & $49(11)$ & 0.005 \\
\hline D-dimer (mg/L) & $0.23(0.23)$ & $0.88(0.60)$ & $<0.001$ \\
\hline $\begin{array}{l}\text { Clopidogrel, } n \\
\text { (\%) }\end{array}$ & $114(21.2)$ & $50(20.9)$ & 0.942 \\
\hline Ticagrelor, $n(\%)$ & $425(78.8)$ & $189(79.1)$ & 0.942 \\
\hline $\begin{array}{l}\text { In-hospital HF, } n \\
(\%)\end{array}$ & $47(8.7)$ & $61(25.5)$ & $<0.001$ \\
\hline
\end{tabular}

DM: diabetes mellitus; WBC: white blood cell; PLT: platelet; Cr: creatinine; TC: total cholesterol; TG: triglyceride; EF: ejection fraction; HF: heart failure.

Moreover, few reports investigated the gender difference in the relationship between D-dimer levels and the incidence of $\mathrm{HF}$ in patients with acute MI.

Thus, the present analysis was performed to evaluate the possible relationship between D-dimer levels and incidence of in-hospital HF in patients with STEMI undergoing pPCI in the overall population and in subgroups with gender difference.

\section{Methods}

2.1. Study Design and Patient Selection. Consecutive patients with STEMI who underwent pPCI at Liaocheng People's Hospital from June 2015 to November 2019 were enrolled in this study. Acute STEMI was defined as follows: chest pain typically lasts more than 30 minutes, and ST-segment elevation of adjacent two electrocardiography leads at least $2 \mathrm{~mm}$ within 12 hours of symptom onset [15]. The symptom onset could be prolonged to 24 hours if evidence of hemodynamic instability or persistent ischemia was existing [10]. Patients diagnosed with STEMI who underwent pPCI with stent implantation were included in the study. The exclusion criteria were as follows: severe infections, severe liver and renal diseases, aortic dissection, immune system diseases, cancer, and severe complications during the procedure. Patients with $\mathrm{HF}$ at admission were also excluded. A total of 778 patients were included in this study. The study flowchart is shown in Figure 1. All the protocols were approved by the Liaocheng People's Hospital's Ethics Committee, and informed consent forms were obtained from all patients.

2.2. Primary PCI. PPCI procedures were performed by experienced cardiologists. All patients received standard loaded dose medications $(300 \mathrm{mg}$ of aspirin and $300 \mathrm{mg}$ clopidogrel or $180 \mathrm{mg}$ ticagrelor) upon the diagnosis of STEMI. After successful puncture, all patients were administrated $2500 \mathrm{IU}$ heparin, with a weight dependent dose (up to $100 \mathrm{IU} / \mathrm{kg}$ ) added for PCI. All decisions and procedures, including radial/femoral approach and intervention strategy, were determined by the operator's discretion.

2.3. Laboratory Testing and Echocardiography. All venous blood samples were collected from patients on admission before the $\mathrm{pPCI}$ procedure. All the laboratory tests were performed by the emergency laboratory of our hospital. The 
TABLE 2: Basic characteristics of patients with gender difference.

\begin{tabular}{|c|c|c|c|c|}
\hline Variable & $\begin{array}{c}\text { All patients } \\
n=778\end{array}$ & $\begin{array}{c}\text { Man } \\
n=582\end{array}$ & $\begin{array}{l}\text { Woman } \\
n=196\end{array}$ & $p$ value \\
\hline Age (years) & $62(17)$ & $59(17)$ & $67(10)$ & $<0.001$ \\
\hline Smoking, $n(\%)$ & $416(53.5)$ & $405(69.6)$ & $11(5.6)$ & $<0.001$ \\
\hline Hypertension, $n(\%)$ & $418(53.7)$ & $305(52.4)$ & $113(57.6)$ & 0.203 \\
\hline $\mathrm{DM}, n(\%)$ & $204(26.2)$ & $138(23.7)$ & $66(33.7)$ & 0.006 \\
\hline Heart rate (bpm) & $76(21)$ & $76(21)$ & $76(20)$ & 0.552 \\
\hline Hemoglobin (g/dL) & $143(21)$ & $147(18)$ & $131(19)$ & $<0.001$ \\
\hline WBC count $(\times 109 / \mathrm{L})$ & $9.8(3.97)$ & $9.95(4.14)$ & $9.55(3.71)$ & 0.083 \\
\hline PLT $(\times 109 / \mathrm{L})$ & $228(77)$ & $224(77)$ & $240(85)$ & 0.025 \\
\hline $\mathrm{Cr}(\mu \mathrm{mol} / \mathrm{L})$ & $62(20)$ & $66(19)$ & $53(17)$ & $<0.001$ \\
\hline $\mathrm{TC}(\mathrm{mmol} / \mathrm{L})$ & $4.70(1.31)$ & $4.64(1.21)$ & $5.10 \pm 1.51$ & $<0.001$ \\
\hline $\mathrm{TG}(\mathrm{mmol} / \mathrm{L})$ & $1.47(1.23)$ & $1.47(1.26)$ & $1.44(1.12)$ & 0.706 \\
\hline $\mathrm{EF}(\%)$ & $50(10)$ & $50(9)$ & $50 \pm 10$ & 0.368 \\
\hline D-dimer (mg/L) & $0.31(0.43)$ & $0.30(0.43)$ & $0.40(0.58)$ & $<0.001$ \\
\hline Clopidogrel, $n(\%)$ & $164(21.1)$ & $115(19.7)$ & $49(25.0)$ & 0.120 \\
\hline Ticagrelor, $n(\%)$ & $614(78.9)$ & $467(80.3)$ & $147(75.0)$ & 0.120 \\
\hline In-hospital HF, $n(\%)$ & $108(13.8)$ & $66(11.3)$ & $42(21.4)$ & $<0.001$ \\
\hline
\end{tabular}

DM: diabetes mellitus; WBC: white blood cell; PLT: platelet; Cr: creatinine; TC: total cholesterol; TG: triglyceride; EF: ejection fraction; HF: heart failure.

TABLE 3: Logistic regression analysis of predictors of in-hospital heart failure in the overall population.

\begin{tabular}{|c|c|c|c|c|c|c|}
\hline Variables & OR & $95 \% \mathrm{CI}$ & $p$ value & Adjusted OR & $95 \% \mathrm{CI}$ & $p$ value \\
\hline Age & 1.033 & $1.014-1.053$ & 0.001 & 1.036 & $1.014-1.059$ & 0.001 \\
\hline Gender & 2.132 & $1.392-3.267$ & 0.001 & 1.101 & $0.612-1.980$ & 0.748 \\
\hline Smoking & 1.816 & $1.201-2.744$ & 0.005 & 1.246 & $0.736-2.112$ & 0.413 \\
\hline Hypertension & 1.627 & $1.068-2.479$ & 0.023 & 1.569 & $1.006-2.448$ & 0.047 \\
\hline Diabetes mellitus & 1.648 & $1.070-2.539$ & 0.023 & 1.484 & $0.936-2.354$ & 0.093 \\
\hline Heart rate & 1.019 & $1.006-1.031$ & 0.003 & 1.022 & $1.009-1.035$ & 0.001 \\
\hline Hemoglobin & 0.982 & $0.971-0.994$ & 0.002 & 0.985 & $0.971-1.000$ & 0.052 \\
\hline WBC count & 1.107 & $1.043-1.175$ & 0.001 & 1.131 & $1.058-1.210$ & $<0.001$ \\
\hline PLT & 1.000 & $0.997-1.003$ & 0.863 & & & \\
\hline $\mathrm{Cr}$ & 1.010 & $0.999-1.021$ & 0.074 & & & \\
\hline TC & 0.880 & $0.721-1.073$ & 0.205 & & & \\
\hline TG & 0.903 & $0.764-1.068$ & 0.234 & & & \\
\hline D-dimer & 1.359 & $1.159-1.594$ & $<0.001$ & 1.197 & $1.003-1.429$ & 0.046 \\
\hline
\end{tabular}

OR: odds ratio; CI: confidence interval; WBC: white blood cell; PLT: platelet; Cr: creatinine; TC: total cholesterol; TG: triglyceride; D-D: D-dimer.

plasma levels of $\mathrm{D}$-dimer were measured by the immunoturbidimetry method using HemosIL D-dimer HS 500 kit (Werfen, Barcelona, Spain) according to the manufacturer's instruction, and $\leq 0.5 \mathrm{mg} / \mathrm{L}$ was defined as normal. Echocardiography was performed within 24 hours on admission to determine left ventricular ejection fraction (LVEF).

2.4. Study Endpoints. Patients were evaluated by an attending cardiologist. According to clinical observations, the definition of HF was mainly based on ESC guidelines for the diagnosis and treatment of acute and chronic HF [16]. HF was defined as the presence of symptoms of breathlessness and clinical signs of pulmonary congestion on physical examina- tion, including an S3 gallop, pulmonary or alveolar rales, and/or interstitial edema during a hospital stay.

2.5. Statistical Analysis. Statistical analysis was performed using the SPSS software, version 23.0 (IBM Corp.). Continuous data with normal distribution were expressed as mean \pm standard deviation and compared using the Student $t$ -test. Data with nonnormal distribution were expressed as median (interquartile range) and compared using the Mann-Whitney $U$ test. Categorical variables were expressed as frequency and percentage and compared using the Chisquare test or Fisher exact test. Logistic regression analysis was used to determine whether D-dimer was an independent 
TABLE 4: Logistic regression analysis of predictors of in-hospital heart failure in the male population.

\begin{tabular}{|c|c|c|c|c|c|c|}
\hline Variables & OR & $95 \% \mathrm{CI}$ & $p$ value & Adjusted OR & $95 \% \mathrm{CI}$ & $p$ value \\
\hline Age & 1.021 & $0.999-1.045$ & 0.065 & & & \\
\hline Smoking & 1.257 & $0.732-2.158$ & 0.406 & & & \\
\hline Hypertension & 1.565 & $0.925-2.650$ & 0.095 & & & \\
\hline Diabetes mellitus & 1.466 & $0.835-2.577$ & 0.183 & & & \\
\hline Heart rate & 1.025 & $1.009-1.041$ & 0.002 & 1.022 & $1.006-1.038$ & 0.007 \\
\hline Hemoglobin & 0.989 & $0.974-1.006$ & 0.200 & & & \\
\hline WBC count & 1.098 & $1.022-1.179$ & 0.011 & 1.072 & $0.995-1.155$ & 0.066 \\
\hline PLT & 1.001 & $0.997-1.004$ & 0.795 & & & \\
\hline $\mathrm{Cr}$ & 1.018 & $1.005-1.032$ & 0.005 & 1.018 & $1.004-1.031$ & 0.010 \\
\hline TC & 0.950 & $0.741-1.218$ & 0.687 & & & \\
\hline TG & 0.929 & $0.763-1.130$ & 0.459 & & & \\
\hline D-dimer & 1.122 & $0.861-1.463$ & 0.395 & & & \\
\hline
\end{tabular}

OR: odds ratio; CI: confidence interval; WBC: white blood cell; PLT: platelet; Cr: creatinine; TC: total cholesterol; TG: triglyceride; D-D: D-dimer.

TABLE 5: Logistic regression analysis of predictors of in-hospital heart failure in the female population.

\begin{tabular}{|c|c|c|c|c|c|c|}
\hline Variables & OR & $95 \% \mathrm{CI}$ & $p$ value & Adjusted OR & $95 \% \mathrm{CI}$ & $p$ value \\
\hline Age & 1.042 & $0.998-1.087$ & 0.061 & & & \\
\hline Smoking & 2.847 & $0.354-22.898$ & 0.325 & & & \\
\hline Hypertension & 1.624 & $0.794-3.322$ & 0.185 & & & \\
\hline Diabetes mellitus & 1.656 & $0.823-3.335$ & 0.158 & & & \\
\hline Heart rate & 1.009 & $0.989-1.031$ & 0.379 & & & \\
\hline Hemoglobin & 0.987 & 0.965-1.009 & 0.251 & & & \\
\hline WBC count & 1.180 & $1.049-1.327$ & 0.006 & 1.143 & $1.009-1.295$ & 0.035 \\
\hline PLT & 0.999 & 0.994-1.004 & 0.652 & & & \\
\hline $\mathrm{Cr}$ & 1.018 & 0.995-1.042 & 0.128 & & & \\
\hline TC & 0.635 & $0.446-0.904$ & 0.012 & 0.671 & $0.461-0.977$ & 0.037 \\
\hline TG & 0.846 & $0.605-1.184$ & 0.330 & & & \\
\hline D-dimer & 1.575 & $1.189-2.086$ & 0.002 & 1.429 & $1.083-1.885$ & 0.012 \\
\hline
\end{tabular}

OR: odds ratio; CI: confidence interval; WBC: white blood cell; PLT: platelets; Cr: creatinine; TC: total cholesterol; TG: triglyceride; D-D: D-dimer.

predictor of in-hospital HF in the total study population, male and female, respectively. Variables such as age, female gender, previous history of smoking, hypertension, and DM were identified with univariate analysis, and the unadjusted $p$ value $<0.05$ in the univariate analysis was subsequently evaluated using a multivariate logistic regression model. Receiver operating characteristic (ROC) curves were used to determine the best cut-off values of $\mathrm{D}$-dimer in predicting in-hospital $\mathrm{HF}$ in the total study population, male and female, respectively. A $p$ value $<0.05$ was considered to be statistically significant.

\section{Results}

3.1. Patient Characteristics. From June 2015 to November 2019, 834 patients underwent pPCI and 778 patients were included in the study finally. Of the 778 patients, 539 $(69.3 \%)$ had normal D-dimer levels $(\leq 0.5 \mathrm{mg} / \mathrm{L})$ and 239 (30.7\%) had increased D-dimer levels $(>0.5 \mathrm{mg} / \mathrm{L})$. The baseline characteristics of the two groups are shown in Table 1. Patients with increased D-dimer levels were older $(p<0.001)$ and nonsmokers $(p=0.032)$ and had a higher incidence of in-hospital HF $(p<0.001)$.

In the subgroup analysis, all patients were divided into two groups according to gender: male (582, 74.8\%) group and female $(196,25.2 \%)$ group (as shown in Table 2$)$. The plasma levels of D-dimer $(p<0.001)$ and the incidence of in-hospital HF $(p<0.001)$ were higher in female patients than in male patients.

3.2. Multivariate Logistic Regression Model for In-Hospital $H F$. For in-hospital HF in the overall population, the result of the multivariate logistic regression revealed that D-dimer was an independent predictor of in-hospital HF in the overall population [adjusted odds ratio (OR): 1.197, 95\% confidence interval (CI): 1.003-1.429, and $p=0.046]$. The results showed that variables such as age, heart rate, and white blood cell (WBC) count were also independent factors of in-hospital HF (Table 3).

For in-hospital HF in the male population, the multivariate logistic regression model variables included heart rate, WBC count, and creatinine. The result revealed that the heart 


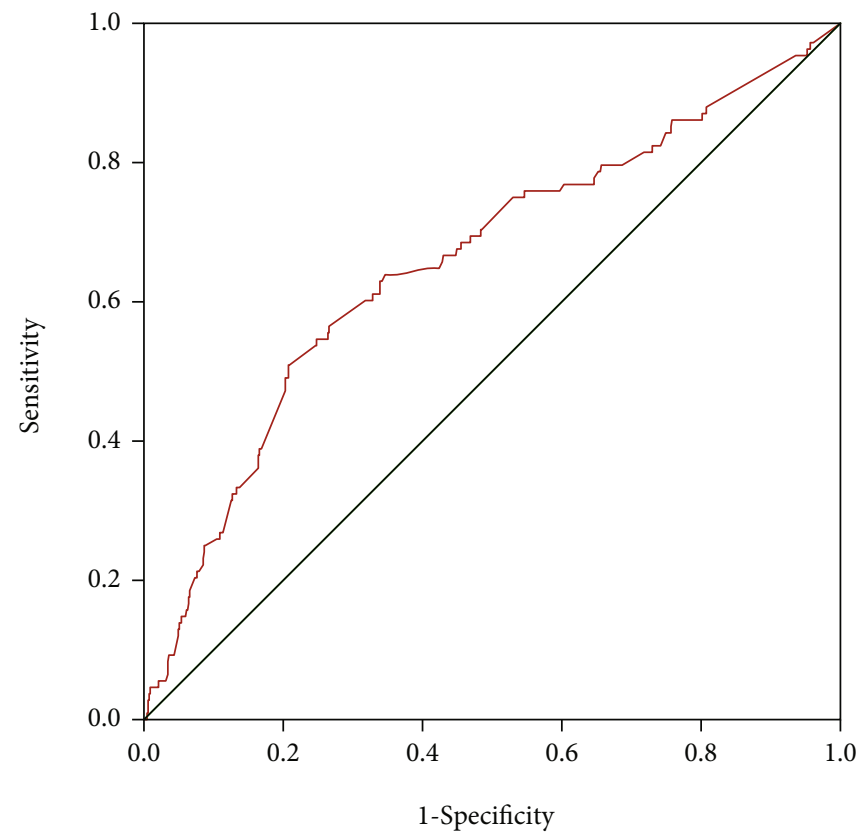

FIgURE 2: Receiver operating characteristic curves of D-dimer for predicting in-hospital heart failure in all patients.

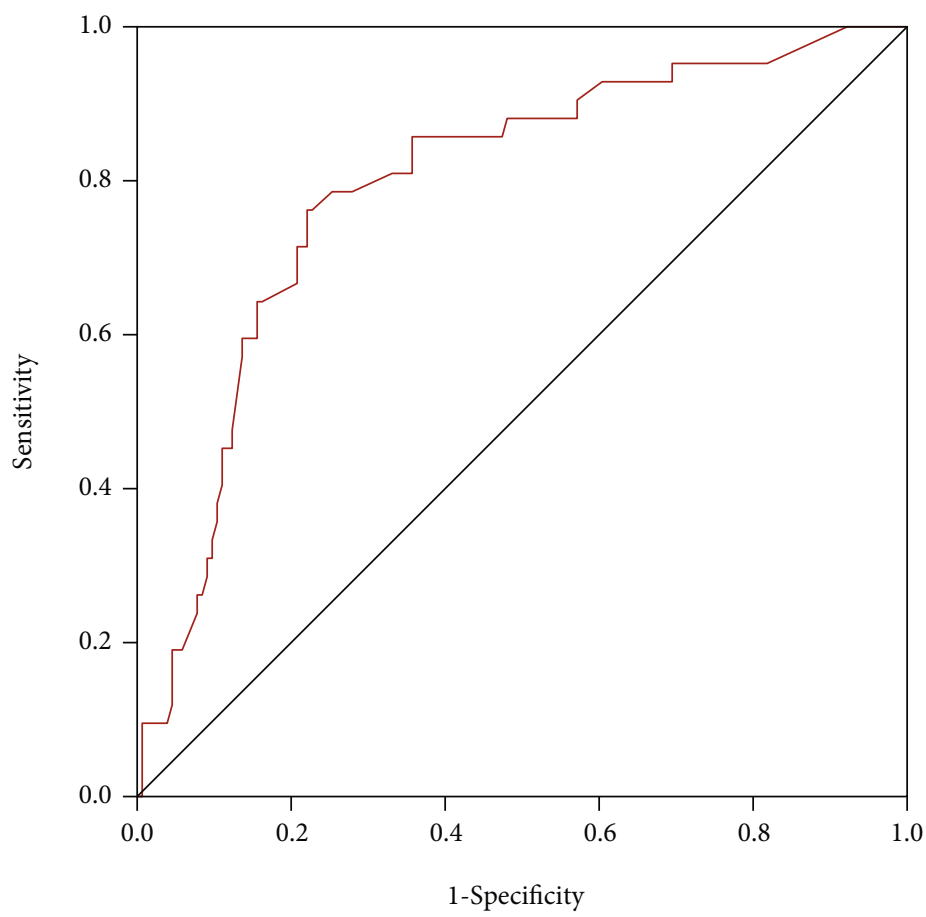

FIGURE 3: Receiver operating characteristic curves of D-dimer for predicting in-hospital heart failure in female patients.

rate, but not D-dimer, was an independent predictor of inhospital HF (Table 4). In the female population, the multivariate logistic regression model included WBC count, total cholesterol (TC), and D-dimer. The result revealed that Ddimer was an independent predictor of in-hospital HF [adjusted OR: $1.429,95 \%$ CI: $1.083-1.885$, and $p=0.012$ ] (Table 5).
3.3. ROC Curves for In-Hospital HF. ROC curves were performed to evaluate the potential predictive power of $\mathrm{D}$ dimer for in-hospital HF in all patients, female and male, respectively (Figures 2-4). The area under the curve (AUC) values for D-dimer in predicting HF were 0.793 with $76 \%$ sensitivity and $78 \%$ specificity $(p<0.001)$ in female patients, 0.657 with $50.9 \%$ sensitivity and $79.3 \%$ specificity $(p<0.001)$ 


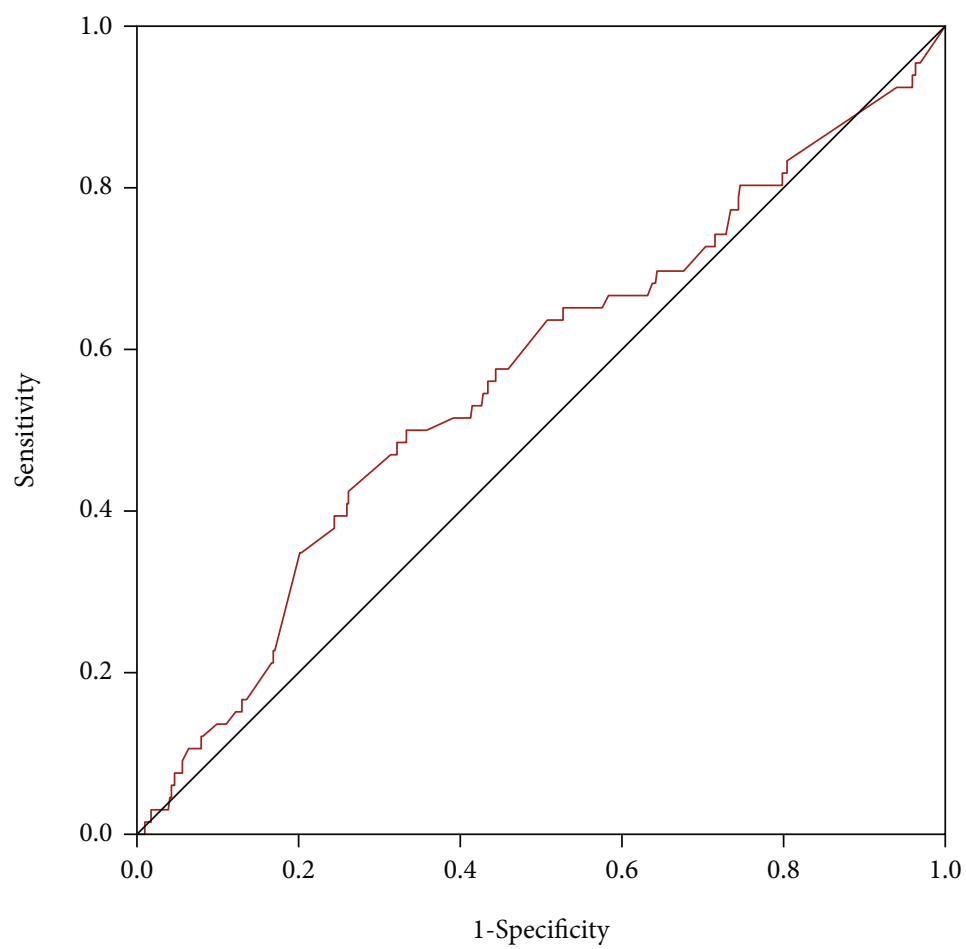

FIGURE 4: Receiver operating characteristic curves of D-dimer for predicting in-hospital heart failure in male patients.

TABLE 6: Performance of gender for D-dimer in prediction in-hospital heart failure.

\begin{tabular}{|c|c|c|c|c|c|c|}
\hline & AUC & $95 \%$ CI & $p$ value & Sensitivity & Specificity & $\overline{\text { Cut-off }}$ \\
\hline Total & 0.657 & $0.597-0.716$ & $<0.001$ & $50.9 \%$ & $79.3 \%$ & 0.615 \\
\hline Male & 0.565 & $0.488-0.643$ & 0.085 & $50 \%$ & $67 \%$ & 0.443 \\
\hline Female & 0.793 & $0.717-0.869$ & $<0.001$ & $76 \%$ & $78 \%$ & 0.615 \\
\hline
\end{tabular}

AUC: area under the curve; CI: confidence interval population.

in the total population, and 0.565 with $50 \%$ sensitivity and $67 \%$ specificity $(p=0.085)$ in male patients. The results of the ROC curve analysis indicated that D-dimer had moderate predictive efficiency for predicting in-hospital HF in female patients and power superior to that in the total population and male patients. The performances of $\mathrm{D}$-dimer in predicting in-hospital HF were shown in Table 6.

\section{Discussion}

This prospective study found that the D-dimer level was an independent predictor of in-hospital HF in patients with STEMI who underwent pPCI. In the subgroup analysis, Ddimer was an independent risk factor of in-hospital HF in female patients but not in male patients.

Several studies evaluated the value of D-dimer in predicting adverse outcomes in patients with coronary artery disease (CAD). The most robust evidence from the LIPID study demonstrated that D-dimer levels could independently predict long-term major cardiovascular mortality in patients with stable CAD [8]. The data from the AtheroGene study unearthed that the $\mathrm{D}$-dimer level was an independent predic- tor of subsequent cardiovascular death in patients with CAD [14]. Moss et al. [17] uncovered that the elevated levels of Ddimer contributed independently to recurrent coronary events in postinfarction patients. Moreover, accumulating evidence has shown that the elevated levels of D-dimer can predict adverse outcomes including death in patients with acute coronary syndrome (ACS) $[6,10,18,19]$. Patients with acute ischemic cerebrovascular disease complicating CHD, whose D-dimer levels were higher than the normal standard, had the worst outcomes [20]. These results were equally indicated that higher levels of D-dimer were associated with a higher incidence of major adverse cardiovascular events (MACEs). However, a retrospective study on 569 patients with STEMI, who underwent pPCI and were prospectively followed up for a median of 38 months, showed that Ddimer has no independent prognostic value in patients with STEMI [21]. Thus, the value of D-dimer in predicting adverse outcomes for CAD was still controversial. Furthermore, the association between $\mathrm{D}$-dimer levels and the incidence of $\mathrm{HF}$ was not explored in the aforementioned studies. Recently, D-dimer was found to be associated with the incidence of HF after hospitalization in patients with 
AMI [22]. Furthermore, in the subsequent LIPID HF riskprediction model, the results revealed that a high level of D-dimer was an independent predictor of incident HF in patients with ACS [23]. More importantly, one multicenter study showed that D-dimer levels were associated with the incidence of HF in adults from the general population [24]. Briefly, the present study results were in accordance with the findings of previous studies, and our study first time revealed the association in the $\mathrm{pPCI}$ population and further elucidated the gender-related difference in D-dimer predicting HF. Further analysis with longer follow-up might help clarify whether high D-dimer levels are an independent predictor of MACEs, especially HF.

A previous study showed that increased levels of D-dimer were significantly associated with myocardial necrosis and inversely correlated with LVEF after AMI [25]. Also, high D-dimer levels on admission were associated with a larger myocardial infarct size assessed using magnetic resonance imaging (MRI) data in patients treated with pPCI for STEMI [26]. Furthermore, multiple pathways such as inflammation [27], neurohormonal activation [28], and stagnation and endothelial dysfunction [29] were associated with the occurrence of $\mathrm{HF}$, which also resulted in $\mathrm{D}$-dimer elevation. More important, patients with $\mathrm{HF}$ were at risk of coagulation disorders and vascular thromboembolic events [30]. These mechanisms might explain the value of D-dimer in independently predicting the incidence of HF in STEMI patients undergoing pPCI.

\section{Limitations}

This study had several limitations. It was a single-center study. Although the sample size was relatively large, the study observation time was limited to the length of in-hospital stay; therefore, a long-term prognostic follow-up is warranted. Finally, data such as culprit lesion characteristics, total ischemic time, and procedural details were not collected and analyzed in this study.

\section{Conclusions}

In this prospective cohort study, increased levels of D-dimer were an independent risk factor of in-hospital HF in patients with STEMI who underwent pPCI, especially women. This analysis highlighted the importance of D-dimer and provided guidance for clinicians in identifying patients at high risk of developing $\mathrm{HF}$ and lowering their risk.

\section{Data Availability}

The data used to support the findings of this study are available from the corresponding author upon request.

\section{Conflicts of Interest}

The authors declare that they have no conflict of interest.

\section{Authors' Contributions}

Li Li and Wei Wang contributed equally to this paper, and they are co-first authors.

\section{Acknowledgments}

This work was supported by the Traditional Chinese Medicine Science and Technology Development Plan Project of Shandong Province (no. 2019-0891).

\section{References}

[1] J. I. Weitz, J. C. Fredenburgh, and J. W. Eikelboom, "A test in context: D-dimer," Journal of the American College of Cardiology, vol. 70, no. 19, pp. 2411-2420, 2017.

[2] M. R. Heldner, S. M. Zuurbier, B. Li et al., "Prediction of cerebral venous thrombosis with a new clinical score and D-dimer levels," Neurology, vol. 95, no. 7, pp. e898-e909, 2020.

[3] P. Bockenstedt, "D-dimer in venous thromboembolism," The New England Journal of Medicine, vol. 349, no. 13, pp. 12031204, 2003.

[4] A. L. Komarov, E. P. Panchenko, A. B. Dobrovolsky et al., "Ddimer and platelet aggregability are related to thrombotic events in patients with peripheral arterial occlusive disease," European Heart Journal, vol. 23, no. 16, pp. 1309-1316, 2002.

[5] G. Palareti, C. Legnani, B. Cosmi et al., "Predictive value of Ddimer test for recurrent venous thromboembolism after anticoagulation withdrawal in subjects with a previous idiopathic event and in carriers of congenital thrombophilia," Circulation, vol. 108, no. 3, pp. 313-318, 2003.

[6] B. Sarli, M. Akpek, A. O. Baktir et al., "Impact of D-dimer level on postinterventional coronary flow and in-hospital MACE in ST-segment elevation myocardial infarction," Herz, vol. 40, no. 3, pp. 507-513, 2015.

[7] H. Naruse, J. Ishii, H. Takahashi et al., "Prognostic value of combination of plasma D-dimer concentration and estimated glomerular filtration rate in predicting long-term mortality of patients with stable coronary artery disease," Circulation Journal, vol. 81, no. 10, pp. 1506-1513, 2017.

[8] J. Simes, K. P. Robledo, H. D. White et al., "D-dimer predicts long-term cause-specific mortality, cardiovascular events, and cancer in patients with stable coronary heart disease: LIPID study," Circulation, vol. 138, no. 7, pp. 712-723, 2018.

[9] X. Zhao, J. Li, X. Tang et al., "D-dimer as a thrombus biomarker for predicting 2-year mortality after percutaneous coronary intervention," Therapeutic advances in chronic disease, vol. 11, article 2040622320904302, 2020.

[10] D. Huang, W. Gao, R. Wu, X. Zhong, J. Qian, and J. Ge, “Ddimer level predicts in-hospital adverse outcomes after primary PCI for ST-segment elevation myocardial infarction," International Journal of Cardiology, vol. 305, pp. 1-4, 2020.

[11] M. C. Bahit, A. Kochar, and C. B. Granger, "Post-myocardial infarction heart failure," JACC: Heart failure, vol. 6, no. 3, pp. 179-186, 2018.

[12] E. Cenko, M. van der Schaar, J. Yoon et al., "Sex-related differences in heart failure after ST-segment elevation myocardial infarction," Journal of the American College of Cardiology, vol. 74, no. 19, pp. 2379-2389, 2019.

[13] X. Zhang, S. Wang, L. Sun, S. Fang, and B. Yu, "Prognostic value of $\mathrm{D}$-dimer in acute myocardial infarction complicated 
by heart failure with preserved ejection fraction," ESC heart failure, vol. 7, no. 6, pp. 4118-4125, 2020.

[14] P. E. Morange, C. Bickel, V. Nicaud et al., "Haemostatic factors and the risk of cardiovascular death in patients with coronary artery disease: the AtheroGene study," Arteriosclerosis, Thrombosis, and Vascular Biology, vol. 26, no. 12, pp. 2793-2799, 2006.

[15] B. Ibanez, S. James, S. Agewall et al., "2017 ESC Guidelines for the management of acute myocardial infarction in patients presenting with ST-segment elevation: the Task Force for the management of acute myocardial infarction in patients presenting with ST-segment elevation of the European Society of Cardiology (ESC)," European Heart Journal, vol. 39, no. 2, pp. 119-177, 2018.

[16] P. Ponikowski, A. A. Voors, S. D. Anker et al., "2016 ESC Guidelines for the diagnosis and treatment of acute and chronic heart failure: the Task Force for the diagnosis and treatment of acute and chronic heart failure of the European Society of Cardiology (ESC) developed with the special contribution of the Heart Failure Association (HFA) of the ESC," European Heart Journal, vol. 37, no. 27, pp. 2129-2200, 2016.

[17] A. J. Moss, R. E. Goldstein, V. J. Marder et al., "Thrombogenic factors and recurrent coronary events," Circulation, vol. 99, no. 19, pp. 2517-2522, 1999.

[18] F. AlKhalfan, M. Kerneis, T. Nafee et al., "D-dimer levels and effect of rivaroxaban on those levels and outcomes in patients with acute coronary syndrome (an ATLAS ACS-TIMI 46 trial substudy)," The American Journal of Cardiology, vol. 122, no. 9, pp. 1459-1464, 2018.

[19] W. J. Kikkert, B. E. Claessen, G. W. Stone et al., "D-dimer levels predict ischemic and hemorrhagic outcomes after acute myocardial infarction: a HORIZONS-AMI biomarker substudy," Journal of Thrombosis and Thrombolysis, vol. 37, no. 2, pp. 155-164, 2014.

[20] Y. Wang, A. Hafeez, F. Meng et al., "The correlation of Ddimer levels with patient outcomes in acute ischemic cerebrovascular disease complicating coronary heart disease," Neurological Research, vol. 38, no. 6, pp. 524-532, 2016.

[21] A. Erkol, V. Oduncu, B. Turan et al., "The value of plasma Ddimer level on admission in predicting no-reflow after primary percutaneous coronary intervention and long-term prognosis in patients with acute ST segment elevation myocardial infarction," Journal of Thrombosis and Thrombolysis, vol. 38, no. 3, pp. 339-347, 2014.

[22] X. Zhang, S. Wang, J. Liu et al., "D-dimer and the incidence of heart failure and mortality after acute myocardial infarction," Heart, vol. 107, no. 3, pp. 237-244, 2021.

[23] A. Driscoll, E. H. Barnes, S. Blankenberg et al., "Predictors of incident heart failure in patients after an acute coronary syndrome: the LIPID heart failure risk-prediction model," International Journal of Cardiology, vol. 248, pp. 361-368, 2017.

[24] R. A. De Boer, M. Nayor, D. F. CR et al., "Association of cardiovascular biomarkers with incident heart failure with preserved and reduced ejection fraction," JAMA Cardiology, vol. 3, no. 3, pp. 215-224, 2018.

[25] C. H. Hansen, V. Ritschel, S. Halvorsen et al., "Markers of thrombin generation are associated with myocardial necrosis and left ventricular impairment in patients with ST-elevation myocardial infarction," Thrombosis journal, vol. 13, p. 31, 2015.

[26] S. Choi, W. J. Jang, Y. B. Song et al., "D-dimer levels predict myocardial injury in ST-segment elevation myocardial infarc- tion: a cardiac magnetic resonance imaging study," PLoS One, vol. 11, no. 8, article e0160955, 2016.

[27] R. Marcucci, A. M. Gori, F. Giannotti et al., "Markers of hypercoagulability and inflammation predict mortality in patients with heart failure," Journal of Thrombosis and Haemostasis, vol. 4, no. 5, pp. 1017-1022, 2006.

[28] S. G. Wannamethee, P. H. Whincup, O. Papacosta, L. Lennon, and G. D. Lowe, "Associations between blood coagulation markers, NT-proBNP and risk of incident heart failure in older men: the British Regional Heart Study," International Journal of Cardiology, vol. 230, pp. 567-571, 2017.

[29] C. O. Hileman, C. T. Longenecker, T. L. Carman et al., "Elevated $\mathrm{D}$-dimer is independently associated with endothelial dysfunction: a cross-sectional study in HIV-infected adults on antiretroviral therapy," Antiviral Therapy, vol. 17, no. 7, pp. 1345-1349, 2012.

[30] B. Greenberg, J. D. Neaton, S. D. Anker et al., "Association of rivaroxaban with thromboembolic events in patients with heart failure, coronary disease, and sinus rhythm: a post hoc analysis of the COMMANDER HF trial," JAMA Cardiology, vol. 4, no. 6, pp. 515-523, 2019. 